\title{
Le design social à l'épreuve Comment démocratiser les politiques de gestion du risque à Sao Paulo?
}

\author{
Jacques Lolive \\ Univ. Grenoble Alpes, CNRS, Science Po Grenoble, PACTE, 38000 Grenoble, France \\ jacques.lolive@umrpacte.fr
}

\section{Résumé}

Dans cet article je présente une recherche-action sur la vie des habitants des zones à risque de la Région Métropolitaine de São Paulo (RMSP) au Brésil menée dans le cadre d'une coopération franco-brésilienne. Dans une première partie j'analyserai les manifestations de la société du risque dans la RMSP et les comportements des habitants des zones à risque. Dans une seconde partie je présenterai notre démarche de design social au service d'un projet de démocratisation des politiques publiques de gestion du risque dans l'État de São Paulo pour améliorer le milieu de vie de ces habitants.

\section{Mots-clés}

Design social, société du risque, habitants des zones à risque, concernement, politiques de gestion du risque, expérience sensible.

\begin{abstract}
Social design test. How to democratize risk management policies in Sao Paulo? - In this article I present a research and action project on the life of the risk areas inhabitants in the São Paulo Metropolitan Region (RMSP) carried in cooperation between France and Brazil. In a first part I will analyze the risk society manifestations in the RMSP and the behaviors of the risk areas inhabitants. In a second part I will present our social design approach for a democratization project of the risk management public policies in the State of São Paulo to improve the living environment of these inhabitants.
\end{abstract}

\section{Keywords}

Social design, risk society, inhabitants of risk areas, concerns, risk management, sensitive experience

\author{
Sommaire / Content \\ 1. Introduction \\ 2. La société du risque remet en question l'habitabilité de la métropole pauliste \\ 3. Comment habiter la société du risque? Expériences d'habitants \\ 4. Des expérimentations pour provoquer le concernement \\ 5. Le design social pour améliorer l'habitabilité des zones à risques \\ 6. Conclusions \\ Bibliographie
}




\section{Ocula ${ }^{20}$}

La place de l'usager en design

J. Lolive I Le design social à l'épreuve I DOI: 10.12977/ocula2019-3

\section{Introduction}

Le design n'est pas seulement l'activité créative capable de concevoir des objets usuels qui soient à la fois beaux et fonctionnels comme une conception trop étroite a pu nous le faire croire autrefois. Pour le professeur de design Alain Findeli, le design n'est pas une profession mais c'est plutôt une attitude selon laquelle ce n'est pas le produit mais « l'être humain qu'il convient de placer au centre du projet en design » (Findeli 2004 : 8). Le design s'intéresse au monde artificiel, construit par l'homme, c'est « le monde comme projet » (Findeli $2004: 12$ ). Plus précisément, le design s'intéresse au rapport qu'entretiennent les êtres humains, concepteurs ou utilisateurs à ce monde, autrement-dit, c'est « le monde pour l'homme », c'est-à-dire le monde comme projet humain à construire ou à préserver et le monde comme projet humain à habiter. Le projet du design vise donc à « maintenir ou améliorer l'habitabilité du monde ». Le design social (cf. Findeli 2016a) est une déclinaison du design qui concerne les projets à forte composante sociale ou sociétale, notamment dans l'espace public. Le « social » du design social renvoie à des projets, notamment dans les pays du sud, qui prennent en charge les besoins et aspirations des populations les plus vulnérables et les injustices dont elles sont victimes. Les autres caractéristiques du design social sont :

- un parti anthropologique qui considère les habitants-usagers comme les porteurs d'un projet d'habiter le monde susceptibles d'émettre des jugements sur la qualité paysagère de leur cadre de vie et soucieux de le voir s'améliorer ;

- le traitement des situations d'incertitude qui sont sources de créativité ;

- la démarche participative qui s'appuie sur l'expérience des populations grâce à la mise en œuvre d'ateliers de co-conception ;

- la modélisation par monstration qui s'appuie sur la conception d'un prototype capable d'incarner la solution satisfaisante et consensuelle d'un ensemble de problèmes.

Un exemple caractéristique de design social est Safe Agua, un programme du Art Center College of Design, situé à Pasadena en Californie, pour faciliter le stockage, l'utilisation, le transport et la conservation d'eau pour les habitants de bidonvilles du Chili. Il a permis de développer des systèmes simples pour l'acheminement de l'eau, sa conservation, ainsi que des systèmes de laveries et de douches communautaires grâce au travail des étudiants du Centre d'art et une institution de bienfaisance de Santiago.

Le design social va fournir une grille interprétative pour redéfinir la cohérence de la recherche que nous menons, ma collègue Cintia Okamura, de la CETESB, l'agence gouvernementale chargée de l'environnement dans l'État de São Paulo, et moi-même. Notre recherche s'inscrit dans la perspective heuristique de la société du risque (cf. Beck 2001) qui considère que le risque est devenu l'horizon indépassable de notre modernité tardive au point de constituer 


\section{Ocula ${ }^{20}$}

La place de l'usager en design

J. Lolive | Le design social à l'épreuve | DOI: 10.12977/ocula2019-3

désormais notre propre milieu de vie. Notre recherche s'appuie sur cette hypothèse pour développer une nouvelle culture du risque, c'est-à-dire pour expérimenter des méthodes participatives qui permettent la prise de conscience du risque par les populations exposées mais expriment également leur droit de vivre aussi bien que possible dans cette société du risque. Comme terrain d'étude, nous avons choisi la Région Métropolitaine de São Paulo (RMSP) au Brésil qui constitue un terrain privilégié d'analyse et d'expérimentation pour une recherche sur les risques. Deux sites d'expérimentation ont été sélectionnés où la population est exposée à des risques complexes : le Condomínio Barão de Mauá (risque de contamination chimique par des substances cancérigènes et risque d'explosion par le méthane) et le terminal pétrolier du port de São Sebastião (risque technologique majeur). Nous avons traduit les connaissances produites par cette recherche dans un protocole qui sera mis en œuvre par la CETESB, notre partenaire institutionnel, et transformera les politiques de gestion des risques dans l'État de São Paulo afin de les ouvrir aux diagnostics des sciences sociales et à la participation des habitants. Notre recherche ne sépare pas l'analyse et la transformation de la situation. C'est une démarche de design social qui vise à rendre plus habitable le milieu de vie des habitants des zones à risque de la RMSP.

L'expérience sensible joue un rôle essentiel dans notre recherche où nous l'associons avec l'enquête pragmatique. La géographie phénoménologique (cf. Dardel 1952, Berque 2000, Hoyaux 2002) analyse l'habiter comme l'humanisation de l'environnement biophysique et objectif par la sphère du symbolique. L'être humain se construit son monde en agençant différents éléments qu'il a prélevé dans l'environnement par des choix perceptifs et d'imagination. Ce processus révèle l'être humain à lui-même grâce aux significations qu'il produit par son faire, son dire, son penser. Ce monde de significations est accessible par l'expérience vécue. Ce courant de la géographie permet de définir l'habiter comme une forme spécifique de rapport à l'environnement. C'est la relation de l'habitant avec son milieu de vie. Le recours à la philosophie pragmatiste (Dewey 1938) permet de redéfinir l'expérience vécue, sensible et affective, de la phénoménologie pour pouvoir mieux l'intégrer dans une démarche de recherche. Pour Dewey, cité par la philosophe Joëlle Zask (2008 : 47), « une expérience consiste à joindre une séquence d'actions à quelque chose que l'on ressent, qui nous affecte ». Cette phase d'examen correspond à une enquête. " Au cours de celle-ci je tâche d'identifier les causes du trouble éprouvé, de les utiliser pour imaginer une hypothèse de résolution, de mettre cette hypothèse à l'épreuve, et ainsi de suite » (idem). Ainsi le ressenti, la séquence affective doit constituer le point de départ d'une enquête pour appartenir à l'expérience. L'enquête ainsi définie est ce que Dewey appelle l'enquête de sens commun, c'est la relation d'adaptation, d'ajustement de nos comportements à l'environnement. Dewey la rapproche de l'enquête scientifique qui obéit à la même structure. Seules les motivations diffèrent. L'enquête de sens commun vise à résoudre des problèmes « d'utilisation et de jouissance » tandis que l'enquête scientifique vise la « connaissance ». Ainsi enquête, expérience et expérimentation sont quasiment synonymes. Pour être véritablement « claire » (c'est-à-dire prétendre au statut d'énoncé scientifique), il faut qu'une idée ait pris en compte les conséquences pratiques qu'elle 
La place de l'usager en design

J. Lolive I Le design social à l'épreuve I DOI: 10.12977/ocula2019-3

est susceptible de produire, ce que seule l'expérimentation peut révéler (Daynac $2002: 3$ ). Lorsque l'environnement est disjoint de l'expérience sensible, il devient inintelligible. La question de l'habitabilité n'est plus prise en compte puisque l'expérience sensible de l'habitant et des petites communautés est gommée. Il faut prendre en compte le rapport sensible au milieu (sensoriel, sensible, imaginatif et signifiant, donc esthétique) exprimé par les habitants, et les riverains pour comprendre la modalité spécifiquement humaine d'adaptation créative à son milieu de vie. Cette relation qui se tisse entre expérience, habitabilité et expérimentation dans une perspective pragmatiste nous permettra de comprendre comment l'on passe du design d'expérience, en prenant en compte l'importance de l'expérience dans l'être humain, au design social en tant que tel, un projet de développement de l'habitabilité d'une communauté vulnérable.

Nous analyserons d'abord la société du risque dans la RMSP et les comportements quotidiens des habitants des zones à risque. L'immersion anthropologique des chercheurs dans une zone contaminée nous permettra de nous appuyer sur l'expérience sensible des habitants pour mieux comprendre les modalités matérielle et esthétique de fabrication et d'entretien du milieu de vie.

Ensuite, nous présenterons notre recherche qui met la méthodologie du design social au service d'un projet de transformation des politiques publiques de gestion du risque pour améliorer le milieu de vie des habitants des zones à risque. Nous expérimenterons en particulier deux méthodes sensibles et participatives, la scène du risque et le théâtre-forum, pour réactiver l'expérience habitante et provoquer le concernement des habitants.

\section{La société du risque remet en question l'habitabilité de la métropole pauliste}

São Paulo, la capitale économique du Brésil est une métropole de 21,5 millions d'habitants (IBGE 2018a). Elle offre toute la modernité disponible avec des shopping-centers luxueux, des entreprises hig-tech et une vie culturelle trépidante. Cependant les inégalités sont extrêmes, dans la ville de São Paulo, les $10 \%$ les plus riches ont des revenus moyens de 22,5 fois plus élevés que les $40 \%$ les plus pauvres. Pour l'État de São Paulo' ${ }^{1}$, l'indice de Palma, le rapport entre la part du revenu approprié pour les 10\% de personnes ayant les revenus les plus élevés et la part appropriée pour les 40\% ayant les revenus les plus bas était de 3,54 en 2017. (IBGE 2018b). Cette situation génère une très forte insécurité. La Région Métropolitaine de São Paulo (RMSP) est une illustration de la société du risque théorisée par Ulrich Beck (2001). Selon lui, la production sociale de richesses est systématiquement corrélée à la production sociale de risques. Ces risques contemporains sont engendrés par la société elle-même ; les sciences et les techniques ne cessant de produire des effets inattendus pouvant être négatifs. Mais São Paulo est une société du risque bien différente de celle des pays développés. Ici, la société du risque

1 Nous ne possédons malheureusement pas les données pour la ville de São Paulo, mais comme les inégalités sont plus fortes dans la capitale, l'indice de Palma y est sans aucun doute plus important. 
La place de l'usager en design

J. Lolive | Le design social à l'épreuve | DOI: 10.12977/ocula2019-3

n'est pas un concept académique. Ce n'est pas la critique réflexive de la société industrielle qui débouche sur une démocratie technique stimulée par les forums hybrides (Callon et al. 2001) où les profanes font des contre-expertises. C'est plutôt le mode de vie des gens dans une métropole inhabitable. Deux exemples :

- Les favelas : 2,16 millions d'habitants de la métropole (IBGE 2011) vivent dans des constructions très précaires sur des aires illégales aux dépends de toute considération de salubrité, d'hygiène ou de sécurité. L'essentiel de la croissance démographique de São Paulo se réalise, se réalisera dans ces périphéries ;

- Les zones contaminées : selon les derniers recensements qui datent de décembre 2017 (CETESB 2017), il y a 2213 zones contaminées dans la seule ville de São Paulo, 3181 dans la RMSP et 5942 dans tout l'État de São Paulo. Des zones d'habitation, des écoles, des universités, des centres commerciaux sont bâtis sur des zones contaminées. On en trouve chaque jour de nouvelles.

São Paulo c'est l'insécurité et le risque au quotidien. Comment vivre dans cette immense métropole où les risques sont omniprésents? Comment habiter cette société du risque?

\section{Comment habiter la société du risque? Expériences d'habitants}

Le Condomínio Barão de Mauá est situé dans la ville de Mauá à $30 \mathrm{~km}$ de São Paulo au Brésil. Composé de 54 bâtiments occupés par 7000 personnes environ, le Condomínio a été implanté en 1996 sur une zone contaminée. La prise de conscience de la contamination a été provoquée par l'explosion causée par le méthane, survenue en avril 2000, qui a tué un ouvrier et brûlé gravement un autre. Depuis cette date, une procédure judiciaire est en cours mais elle s'éternise et les résidents restent exposés au double risque de contamination par des substances cancérigènes comme le benzène, et d'explosion par le méthane. Notre recherche a analysé cette situation en restituant la persistance du milieu de vie dans la zone contaminée. L'immersion anthropologique des chercheurs durant cinq jours dans le condominium a permis de mieux comprendre la manière dont ce territoire à risque était habité et vécu par ses habitants.

Les habitants se font les porte-parole d'une conception spécifique de l'environnement, celle du milieu de vie. Habiter pour l'homme, c'est transformer l'environnement (le donné objectif) d'une manière qui lui soit propre pour en faire son milieu de vie (Berque 2000). Le milieu de vie procède d'une poétique, d'une création esthétique qui s'appuie sur un prélèvement partiel, partial, sélectif, orienté dans l'environnement des humains. Il ne se limite pas au lieu de résidence, il est composé de plusieurs lieux. Ce phénomène s'exerce à différentes échelles : celle de l'individu, du petit collectif, du groupe social, des nationaux, de l'humanité dans son ensemble. Ce milieu humain est constitué par la manière que nous avons d'appréhender les choses par nos sens, nos mots, nos pensées, nos actions. Cette appropriation de l'environnement par les habitants fait l'objet d'une expérience vécue qui est à la fois sensorielle, sensible et imaginative. 


\section{Ocula ${ }^{20}$}

La place de l'usager en design

J. Lolive | Le design social à l'épreuve | DOI: 10.12977/ocula2019-3

Les témoignages des habitants traduisent une perte de confiance dans les repères sensibles. Les odeurs, la vue ne sont plus fiables, les sens des habitants sont abusés. Malgré leur belle apparence, on ne peut pas manger les fruits sur les arbres du condominium. Les entretiens témoignent d'une vigilance accrue. Une odeur diffuse se dégage, plus à certains endroits qu'à d'autres, semble-til, qui inquiète les habitants : s'agit-il d'effluves de méthane ou bien s'agit-il d'effluves de benzène, un gaz cancérigène, ou bien les deux à la fois, ou encore d'un mélange entre tout ça et les odeurs du complexe pétrochimique à proximité? Au niveau sonore, de nombreux habitants gardent en mémoire l'explosion de 2000, si bien que quand ils entendent un bruit soudain, un bruit de choc, ils ne peuvent s'empêcher de penser à l'éventualité d'une autre explosion. Un état de vigilance auditive perdure, comme si les habitants restaient aux aguets d'un accident à venir.

Cependant le milieu de vie persiste dans la zone contaminée. Les entretiens restituent l'effort pour vivre dans cette zone inhospitalière et l'énergie de la vie quotidienne qui continue. La volonté d'habiter le condominium en dépit du risque s'exprime avec force dans les témoignages. La majorité des résidents préfèrent rester dans le condominium parce qu'il est « bien, tranquille et sécure ». La persistance du milieu de vie s'exprime également à travers la solidarité qui se manifeste dans leur mobilisation pour obtenir une réparation de la part des entreprises qui ont contaminées le terrain, construit les immeubles et commercialisé les logements en toute connaissance de cause.

Une caractéristique de l'habitat dans le condominium qui ne se retrouve pas dans les autres zones d'habitation de Mauá, c'est l'aménagement des espaces communs qui évoque le Home sweet home anglo-saxon. En témoignent les pelouses impeccablement tondues en bas des bâtiments, les bancs blancs en fer forgé décoratif qui les agrémentent sans oublier les petits animaux en plâtre inspirés de Walt Disney et les petits panneaux aux couleurs pastel avec des messages lénifiants comme « Dieu bénit cette maison » ou « Le bonheur habite ici ». Cela peut nous sembler le triomphe du kitsch mais la mobilisation de cette esthétique à la portée de toutes les bourses et de toutes les consciences permet d'installer dans la zone contaminée l'utopie du bien-être paisible, un milieu chargé de sens pour la plupart des habitants du condominium. Puisque la zone contaminée menace le milieu de vie des résidents, la production du Home sweet home vise à recréer dans cette même zone un lieu aménagé et approprié qui prolonge le logement et protège les habitants. Les caractéristiques de ce milieu de vie humanisé, chaleureux, bien tenu, saturé de sens, empreint de sentimentalité, s'opposent en effet point par point à celles de l'espace contaminé, désolé, hostile, dangereux et dépourvu de sens qu'il s'agit de tenir à distance. Cette petite bulle protectrice (cf. Sloterdijk 2002) offre un fragile rempart esthétique, imaginaire et sensible à la contamination du milieu de vie.

Ainsi la zone pilote du Condomínio Barão de Mauá nous offre une situation favorable à une démarche de design social, les enjeux d'habitabilité y sont exacerbés et un projet spontané de design par les habitants s'esquisse pour améliorer l'habitabilité de leur milieu de vie. 


\section{Ocula ${ }^{20}$}

La place de l'usager en design

J. Lolive | Le design social à l'épreuve | DOI: 10.12977/ocula2019-3

\section{Des expérimentations pour provoquer le concernement}

Le design d'expérience « s'appuie sur une conception de l'être humain globale conforme à la réalité de l'expérience humaine » (Findeli 2016b : 3). Les deux méthodes que nous allons présenter illustrent cette modalité du design.

La première méthode, le théâtre-forum, permet de faire l'expérience imaginaire d'une catastrophe. São Sebastião est une ville de 81718 habitants située à $200 \mathrm{~km}$ à l'Est de la ville de São Paulo. La ville accueille le plus grand terminal pétrolier d'Amérique latine, connu sous le nom de TEBAR, qui reçoit environ $50 \%$ de tout le pétrole qui arrive dans le pays. Cet énorme terminal, propriété de l'entreprise PETROBRAS, est situé en pleine ville. Les immenses cuves de TEBAR se partagent l'espace avec trois quartiers d'habitation (Vila Amelia, Porto Grande et Topolândia), le centre historique et le centre commercial de la ville. Jusqu'à présent, l'entreprise n'a pas préparé les riverains du terminal à la survenue possible d'un événement grave, il est donc très difficile pour eux d'imaginer ce que serait une possible catastrophe qui métamorphoserait cette agréable cité balnéaire du littoral nord en un véritable enfer. En accord avec l'entreprise, nous avons transposé la méthode du théâtre-forum crée par le dramaturge brésilien Augusto Boal pour construire une situation dans laquelle les habitants du quartier de Vila Amelia enclavé dans le terminal vont faire l'expérience sensible et imaginaire d'une catastrophe simulée. Avec l'aide de l'entreprise et des institutions concernées, nous avons défini un scénario de risque : un incendie se déclare dans une cuve de pétrole à l'intérieur du terminal. Ce scénario est mis en scène par des acteurs qui interpellent le public composé des habitants et des institutions gestionnaires du risque pour qu'il participe à un débat. Ce scénario est la simulation d'une catastrophe susceptible d'impliquer le public grâce à la mise en scène théâtrale. Elle diffère en cela d'une simulation basée sur une communication rationnelle trop souvent vécue comme une simple formalité. Les savoir-faire artistiques recréent une " ambiance de catastrophe » afin de rendre présente la catastrophe, sans qu'elle ait lieu, pour sensibiliser la population exposée. Cinq scènes représentent différentes situations plausibles qui questionnent des membres des institutions de la cité et des habitants :

- Un fils essaie de lever sa mère âgée et alitée pour se conformer à la demande d'évacuer le quartier ;

- La professeure d'une école ne sait pas comment retirer les élèves de l'école et où aller;

- Les voitures sont bloquées par un énorme embouteillage, un camion chargé d'un produit pour étouffer l'incendie tente d'arriver à TEBAR, mais les voitures lui barrent la route ;

- Une mère a perdu son enfant qui était sous la responsabilité de l'école ;

- Une professeure ignore quelles substances sont stockées dans les cuves du terminal et ce qui pourrait arriver si une cuve prend feu.

Cette simulation a permis que les institutions prennent conscience de leur manque de préparation pour faire face aux situations mises en scène. Elle a suscité aussi un fort désir des habitants qui participaient d'être associés à notre démarche. Le théâtre-forum a fonctionné comme un design d'expé- 
La place de l'usager en design

J. Lolive | Le design social à l'épreuve | DOI: 10.12977/ocula2019-3

rience. Grâce à lui, le public a vécu par l'imagination cette expérience d'une catastrophe qui permet de mieux se préparer à l'événement et d'améliorer les dispositifs existants.

Avec la seconde méthode, la scène du risque, l'expression publique des émotions permet aux habitants de se réapproprier leur milieu de vie. Nous retournons dans le Condomínio Barão de Mauá, cette zone contaminée où les habitants sont exposés aux risques de contamination et d'explosion depuis 20 ans! L'explosion survenue en avril 2000 a constitué un véritable traumatisme pour les habitants et leur désarroi s'exprime avec force face à une situation douloureuse et bloquée. La vente en 1995 du terrain contaminé par l'entreprise COFAP, avec l'approbation de la mairie, pour y construire le futur condominium suscitera plus tard l'indignation morale des habitants devant le manque de respect des institutions à leur égard. Cette indignation rétrospective s'exprimera lors de la prise de conscience de la contamination après l'explosion. Elle a favorisé l'essor rapide du mouvement des habitants. Ce contexte pesant et chargé d'affects a également accompagné notre recherche tout au long de sa progression. Lors de la semaine d'immersion, notre équipe de recherche avait prévu d'organiser un atelier de réactivation photographique qui proposait aux habitants de commenter un corpus photographique du site (26 photographies choisies par l'équipe de recherche) pour servir de base à la discussion. La séance s'est transformée en une réunion publique. Les participants ont évoqué leurs souffrances : le traumatisme de ceux qui ont assisté à l'accident, la terreur de vivre ici au milieu du danger ; la stigmatisation dont ils sont l'objet. Durant les deux séances de l'atelier de réactivation, ces émotions ont soutenu des critiques très vives des institutions, particulièrement de la CETESB qui a déçu les attentes des habitants à son égard. Nous sommes nous-mêmes interpellés et critiqués parfois avec véhémence parce qu'une partie des membres de l'équipe sont de la CETESB. Selon nos analyses, les habitants qui nous ont imposé cette situation ont exprimé leur besoin de disposer d'une scène du risque, c'est-àdire d'un lieu et d'occasions consacrés à l'expression publique des émotions.

Je reprends ici le terme des sociologues Geneviève Decrop, Christine Dourlens et Pierre Vidal-Naquet (Decrop et al. 1997) mais avec une signification bien différente. L'hypothèse de ces auteurs au début de leur recherche était que les risques naturels s'appréhendaient à travers des négociations entre différents types d'acteurs dans l'espace local. Ils espéraient trouver dans leurs études de cas une tendance à « la gestion négociée des risques avec une confrontation des points de vue ou des intérêts à travers un débat démocratique visant la transparence » (idem : 8). D'où leur choix de la notion de scènes locales pour exprimer ces discussions sous le regard du public. Cependant, de leur propre aveu, ce que nos auteurs ont trouvé sur le terrain n'avait qu'un rapport assez lointain avec cette modalité de démocratie participative. « D'une manière générale, ce que l'on voyait à l'œuvre, c'était davantage des transactions, menées à bas bruit et hors des feux de la rampe que des négociations au sens formel de ce mot » (ibid. : 8). Cette opacité des scènes locales $\mathrm{du}$ risque s'expliquait d'après les auteurs par le fait que s'y jouait l'acceptation de certains types de risques, comme l'inondation, et des dommages qu'il étaient susceptibles de causer à certaines catégories sociales exclues de 


\section{Ocula ${ }^{20}$}

La place de l'usager en design

J. Lolive | Le design social à l'épreuve | DOI: 10.12977/ocula2019-3

la protection. Même si le risque zéro n'existe pas, on conçoit que ce renoncement à protéger tous les citoyens et ce choix d'exclure certains puissent difficilement faire l'objet d'un débat public. Cette recherche révèle ainsi la disjonction qui existe entre un problème, dramatiquement vécu par les gens affectés, et son traitement dans les forums, qui en débattent. Nous partageons la conclusion de Decrop pour qui la raison publique ne se réduit pas à la rationnalité technico-scientifique et les affects, les valeurs ne peuvent être rejetées dans l'irrationnel. Nous distinguerons donc le forum où l'on tente de débattre démocratiquement de l'existence du risque et de sa gestion d'avec la scène du risque consacrée à l'expression collective des affects et des valeurs pour souligner comment la procédure démocratique classique est déconnectée de la production de ce qui fait sens et nous les unirons dans un dispositif complémentaire pour expérimenter les effets d'une injection d'affects dans une procédure démocratique.

En effet, si l'on se réfère à l'amélioration des relations avec les habitants que nous avons pu constater ensuite, cette scène du risque improvisée a permis d'apaiser l'expérience douloureuse des riverains par la mise en commun des émotions et une forme de reconnaissance par autrui censée réparer l'identité morale blessée par le manque de respect. Cette circulation des émotions nous a permis de mieux comprendre le fait qu'un territoire du risque, un site contaminé, affecté, pollué reste un milieu de vie. Le considérer en tant que tel pourrait favoriser une réappropriation du site par ses habitants et une prise en charge collective. Ce dispositif de la scène du risque, qu'il se mette en place spontanément ou qu'il soit provoqué par une ingénierie participative, correspond aussi à un design d'expérience puisque l'expression collective des émotions rappelle l'expérience de la contamination.

Ces deux design d'expérience nous ont permis de mieux comprendre comment l'habitant des zones à risque affecté par les transformations de son milieu de vie pourrait se transforme en un porteur de projet collectif de design social. La notion de concernement (cf. Brunet 2008) décrit ce processus qui produit un potentiel d'engagement, une disponibilité pour la mobilisation. Si nous transposons au design, la notion définira toute sensibilité de l'habitant orientée vers son milieu de vie lorsque des transformations importantes, imposées et subies de ce milieu menacent son habitabilité et qui s'exprime par un comportement plus ou moins actif susceptible de prendre place dans un processus visant à améliorer cette habitabilité.

L'expérimentation des méthodes nous a permis d'analyser quelques modalités de fonctionnement du concernement. Nos deux terrains de recherche sont des lieux de résidence devenus zone à risque et zone contaminée. Ces transformations importantes, subies, imposées par de grandes entreprises ou institutions sont considérées par les habitants comme des atteintes intolérables à leur milieu de vie d'autant plus qu'elles concentrent leurs effets sur leur lieu de résidence qui constitue la localité protectrice par excellence. Elles vont produire une sensibilité réactive, un affect qui exprime les relations matérielles et non-matérielles de l'habitant à son milieu de vie menacées par les transformations. Il révèle les attachements de l'habitant, ce à quoi il tient et ce qu'il craint de perdre. C'est cette modalité de l'expérience habitante que nous 
La place de l'usager en design

J. Lolive | Le design social à l'épreuve | DOI: 10.12977/ocula2019-3

analysons et expérimentons avec nos méthodes. Elles fonctionnent comme l'esthétique relationnelle (Bouriaud 2001) qui installe des relations sociales pour permettre de nouvelles possibilités de vie. Avec nos méthodes, une performance esthétique installe une situation dans laquelle les gens vont faire une expérience esthétique qui résonne avec une expérience habitante. Pour expliquer cette résonnance, nous définirons la correspondance qui la rend possible. Le tableau qui suit résume ces analyses.

Tableau 1. Fonctionnement du concernement.

\begin{tabular}{|c|c|c|c|c|}
\hline & $\begin{array}{l}\text { Performance } \\
\text { esthétique }\end{array}$ & $\begin{array}{l}\text { Expérience } \\
\text { esthétique }\end{array}$ & $\begin{array}{l}\text { Correspon- } \\
\text { dance }\end{array}$ & $\begin{array}{l}\text { Expérience } \\
\text { habitante }\end{array}$ \\
\hline Scène du risque & $\begin{array}{l}\text { Mise en scène } \\
\text { «théâtrale » : } \\
\text { auto-présenta- } \\
\text { tion d'une popu- } \\
\text { lation exposée à } \\
\text { la contamination } \\
\text { qui met en scène } \\
\text { ses émotions }\end{array}$ & $\begin{array}{l}\text { Expression } \\
\text { publique et mise } \\
\text { en commun des } \\
\text { émotions habi- } \\
\text { tantes suscitées } \\
\text { par la vie dans } \\
\text { une zone conta- } \\
\text { minée } \\
\text { Partage du } \\
\text { sensible et des } \\
\text { émotions }\end{array}$ & $\begin{array}{l}\text { Concernement : } \\
\text { les affects } \\
\text { (pré-personnels } \\
\text { et préconscient) } \\
\text { révèlent les } \\
\text { modifications } \\
\text { du milieu de vie } \\
\text { et spécialement } \\
\text { le milieu de vie } \\
\text { affecté }\end{array}$ & $\begin{array}{l}\text { L'expérience } \\
\text { de la contami- } \\
\text { nation } \\
\text { Une mémoire } \\
\text { des pratiques } \\
\text { d'habitants } \\
\text { marquées par } \\
\text { leur vulnérabili- } \\
\text { té et celle de leur } \\
\text { milieu de vie }\end{array}$ \\
\hline Théâtre-forum & $\begin{array}{l}\text { Mise en scène } \\
\text { théâtrale : un } \\
\text { réalisateur met } \\
\text { en scène une } \\
\text { catastrophe } \\
\text { potentielle }\end{array}$ & $\begin{array}{l}\text { Faire l'expé- } \\
\text { rience sensible } \\
\text { et imaginaire } \\
\text { d'une catas- } \\
\text { trophe simulée } \\
\text { pour se préparer } \\
\text { à la catastrophe } \\
\text { réelle }\end{array}$ & $\begin{array}{l}\text { Simulation : } \\
\text { impliquer les } \\
\text { spectateurs dans } \\
\text { une scène de } \\
\text { catastrophe pour } \\
\text { leur révéler leur } \\
\text { implication dans } \\
\text { une situation } \\
\text { réelle de risque } \\
\text { catastrophique }\end{array}$ & $\begin{array}{l}\text { «L'expérience » } \\
\text { de la catastrophe } \\
\text { Une antici- } \\
\text { pation des } \\
\text { pratiques dans } \\
\text { une situation de } \\
\text { catastrophe }\end{array}$ \\
\hline
\end{tabular}

Des mécanismes particuliers vont assister ce mécanisme général :

- La mise en scène de l'expérience par le théâtre ;

- L'expression publique des émotions qui permet de publiciser les affects ressentis quand le milieu de vie est affecté ;

- L'imagination qui permet de se dégager de l'inertie et la myopie du présent pour expérimenter la catastrophe et apprécier sa dangerosité.

Ces analyses du concernement reproduit par une ingénierie participative permettent de comprendre quelques mécanismes du concernement spontané qui transforment les habitants pour les rendre disponibles à certaines modalités d'engagement, comme les mobilisations dans l'espace public ou la participation à un projet de design social.

\section{Le design social pour améliorer l'habitabilité des zones à risque}

Dans ce chapitre, je présenterai notre démarche de design social pour transformer les politiques publiques de gestion du risque afin d'améliorer la situation des habitants des zones à risque de la RMSP. C'est une méthodologie de modélisation pragmatique. Le modèle fonctionne ici comme un cadre d'interactions réelles qui permet d'expérimenter certaines hypothèses 


\section{Ocula ${ }^{20}$}

La place de l'usager en design

J. Lolive | Le design social à l'épreuve | DOI: 10.12977/ocula2019-3

de transformations à l'échelle d'un site pilote pour ensuite mettre en œuvre les résultats de cette expérimentation dans l'action publique.

La modélisation du phénomène complexe recouvre trois opérations. 1) La modélisation simplifie le phénomène étudié, ici l'exposition aux risques, pour que l'on puisse intervenir dessus. C'est décrire le réseau des acteurs principaux et leurs logiques d'action qui participent à la production du phénomène avec des hypothèses de fonctionnement et de transformation. Ainsi le diagnostic réalisé sur le Condomínio Barão de Mauá nous a révélé l'existence de deux réalités très différentes : le monde des politiques publiques relativement clos sur lui-même, où les actions sectorielles des institutions s'entrechoquent l'une l'autre sans qu'il y ait de véritables échanges ni de volonté de collaboration, et le monde vécu des habitants qui sont tiraillés entre la prise en compte contraignante du risque et l'aspiration au retour à la normale. 2) La mise en place d'un cadre d'interaction qui simule les interrelations plus complexes de la société permet d'expérimenter les transformations au sein du modèle. Ainsi, la mise en place d'un dispositif de participation composé de deux forums complémentaires qui sont appelés à travailler ensemble, l'un composé des habitants du condominium et l'autre des différentes institutions concernées, nous permet d'expérimenter une gestion participative et partenariale du risque pour débattre des problèmes communs et définir des solutions consensuelles. On retrouve ici la modélisation par monstration et prototypage du design social. 3) L'expérimentation à petite échelle dans des sites pilote représentant un territoire plus complexe permet de contextualiser, d'analyser les effets du contexte sur l'action. Comme une bonne partie de la population métropolitaine habite dans une zone contaminée ou dans le voisinage immédiat d'une industrie dangereuse, nous avons choisi deux sites pilotes représentant ces situations de risque emblématiques de la métropole.

Nos résultats de recherche vont être mis en œuvre dans l'action publique afin de transformer la situation des habitants. Notre objectif est d'enrichir les politiques publiques de gestion du risque pour qu'elles s'inspirent des méthodes, analyses et problématiques des sciences sociales et s'ouvrent davantage au milieu anthropique et à ses habitants, ce qui suppose un renforcement de leur caractère participatif. Ces orientations sont inscrites dans notre protocole de communication participative du risque qui sera mis en œuvre par la CETESB et s'imposera aux institutions et aux entreprises. Comment transformer ce protocole pour qu'il puisse être utilisé par des ingénieurs et des fonctionnaires d'une administration? Le premier objectif est de parler un langage compréhensible pour les ingénieurs. Pour y parvenir nous devons formaliser au maximum les données qualitatives recueillies dans notre recherche pour les opérationnaliser, c'est-à-dire pour les mettre au service de la décision. Le second objectif est plus difficile puisqu'il s'agit de modifier la culture d'entreprise de la CETESB dominée par les ingénieurs. Plutôt que de se risquer à un débat sur le plan des valeurs, nous proposons des recettes pour créer des habitudes vertueuses. Nous sommes en train de prolonger le protocole par un roteiro, un mode d'emploi qui guide pas à pas l'ingénieur et le cadre d'entreprise dans la mise en ouvre du protocole. Enfin, nous utiliserons la participation des habitants pour faire entendre leurs perceptions et leurs attentes et faire pression sur les ingénieurs. 


\section{Ocula ${ }^{20}$}

La place de l'usager en design

J. Lolive | Le design social à l'épreuve | DOI: 10.12977/ocula2019-3

\section{Conclusions}

La conception large du design proposée dans le monde anglo-saxon et notamment nord-américain redéfinit l'aménagement comme un projet participatif d'amélioration de l'habitabilité du monde. Cette perspective nous a permis de redéfinir notre recherche sur la société du risque dans la Région Métropolitaine de São Paulo (RMSP) comme un projet de design social s'appuyant sur l'analyse d'une situation singulière, la vie difficile des habitants de la zone contaminée du Condomínio Barão de Mauá, pour améliorer leur situation. Comparable au design d'expérience, notre recherche s'appuie essentiellement sur le recueil de l'expérience des habitants pour mieux comprendre la manière dont ce territoire à risque était habité et vécu par ses habitants et pour restituer leurs efforts multiformes pour préserver l'habitabilité de leur milieu de vie. Dans cette même logique, nous expérimentons des méthodes, théâtre-forum et scènes du risque, qui réactivent cette expérience pour susciter le concernement des habitants afin de les impliquer dans notre démarche de design social participatif qui vise à transformer les politiques publiques de gestion du risque afin d'améliorer la situation des habitants des zones à risque de la RMSP. Interpréter notre recherche comme un projet de design social nous a permis de mieux comprendre son mode de fonctionnement à partir de la notion de modélisation. Notre démarche peut ainsi s'analyser comme une modélisation originale dans laquelle la mise en place d'un cadre d'interactions simplifiées mais réelles nous permet d'expérimenter des hypothèses de transformations à l'échelle du site pilote pour ensuite mettre en œuvre les résultats de cette expérimentation dans l'action publique.

Comment comprendre l'efficacité de cette transposition du design social dans le champ de la science politique et quelles sont ses limites? Je propose une explication provisoire. Le design social peut s'analyser comme une redéfinition de la politique à partir du planning, en particulier de l'advocacy planing (cf. Davidoff 1965). Il transforme la politique publique en un projet d'ingénierie sociale conduit par un groupe de concepteurs mais ce n'est pas une politique technocratique classique puisque l'ingénierie sociale est animée par des valeurs progressistes comme l'humanisme, la participation et la justice sociale. Ainsi le planning social pourrait fournir un cadre à la démocratisation des politiques de gestion du risque parce qu'il valorise la participation des populations exposées aux risques et parce qu'il tente de susciter un concernement de l'habitant pour qu'il s'engage dans une procédure, une politique publique ou une mobilisation. C'est sans doute parce qu'il est étranger aux présupposés de la théorie politique qu'il peut nous aider à comprendre ce fondement de la politique ancré dans l'expérience de l'habitant. Peut-il se substituer pour autant à la sociologie politique pour analyser les mobilisations habitantes qui débordent les procédures, même les plus démocratiques? Je ne le pense pas car sa méconnaissance des catégories de l'action collective et des mobilisations constitue alors une faiblesse. Il convient donc d'articuler cette perspective stimulante du design social avec des analyses de la mobilisation inspirées par la sociologie politique. 


\section{Ocula ${ }^{20}$}

La place de l'usager en design

J. Lolive | Le design social à l'épreuve | DOI: 10.12977/ocula2019-3

\section{Bibliographie}

Beck, Ulrich

2001 La société du risque, Paris, Aubier.

Berque, Augustin

2000 Écoumène. Introduction à l'étude des milieux humains, Paris, Belin.

Brunet, Philippe

2008 « De l'usage raisonné de la notion de « concernement » : mobilisations locales à propos de l'industrie nucléaire ", Natures Sciences Sociétés, Vol. 16, n 4 , pp. $317-325$.

Callon, Michel ; Lascoumes, Pierre ; Barthe, Yannick

2001 Agir dans un monde incertain. Essai sur la démocratie technique, Paris, Le Seuil.

CETESB - Companhia Ambiental do Estado de São Paulo

2017 Relação de áreas contaminadas, São Paulo, décembre.

Dardel, Éric

1952 L’Homme et la Terre : nature de la réalité géographique, Paris, PUF (réédition Paris, Editions du CTHS, 1990).

Davidoff, Paul

1965 « Advocacy and Pluralism in Planning », Journal of the American Institute of Planners, Vol. 31, $\mathrm{n}^{\circ}$ 4, pp. 331-338.

Daynac, Michel

2002 Pragmatisme, expertise et énoncés scientifiques. Réflexions sur l'utilisation de l'expertise dans la formulation des énoncés scientifiques en sciences sociales, <http://www.afscet.asso.fr/resSystemica/Creteo2/Daynac.pdf>.

Decrop, Geneviève et al.

1997 Les scènes locales du risque, Rapport final, recherche financée par le Contrat de Plan État Région Rhône-Alpes, programme Risques naturels et Génie urbain et environnement, <http://www.risknat.org/pages/programme_dep/ docs/futur_anterieur/1995_Decrop-1997-final.pdf>.

Dewey, John

1938 Logic : the theory of inquiry, New York : Henry Holt and Company ; trad. fr. Logique. La théorie de l'enquête, Paris, PUF 1993.

Findeli, Alain

2004 « La recherche-projet : une méthode pour la recherche en design », communication présentée au premier Symposium de recherche sur le design tenu à la HGK de Bâle sous les auspices du Swiss Design Network les 13-14 mai 2004, <projekt.unimes.fr/files/2014/04/Findeli.2005.Recherche-projet.pdf

2016a « Le design social », communication présentée à la journée d’études Innover! Innover! Oui mais comment? Ce que nous apporte le Design Social, Société Française d'Évaluation, 24 mars, Nîmes, <http://www.sfe-asso.fr/sites/default/files/document/le_design_social_par_alain findeli.pdf $>$.

2016b Synthèse de la journée d'études Innover! Innover! Oui mais comment? Ce que nous apporte le Design Social, Société Française d'Évaluation, 24 mars, 


\section{Ocula ${ }^{20}$}

La place de l'usager en design

J. Lolive | Le design social à l'épreuve | DOI: 10.12977/ocula2019-3

Nîmes, <http://www.sfe-asso.fr/sites/default/files/document/16-3-24_synthese_seminaire_design_vdef.pdf $>$.

Hoyaux, André-Frédéric

2002 « Entre construction territoriale et constitution ontologique de l'habitant : Introduction épistémologique aux apports de la phénoménologie au concept d'habiter », Cybergeo : European Journal of Geography, <http://journals. openedition.org/cybergeo/1824>.

IBGE (Instituto Brasileiro de Geografia e Estatistica)

2011 Censo demográfico 2010. Aglomerados subnormais. Primeiros resultados, Ministério do Planejamento, Orçamento e Gestão, Rio de Janeiro.

2018a Estimativas da população residente nos municípios brasileiros com data referência em $1^{\circ}$ de julho de 2018, Ministério do Planejamento, Orçamento e Gestão, Rio de Janeiro.

2018b Síntese de Indicadores Sociais. Uma análise das condições de vida da população brasileira, Ministério do Planejamento, Orçamento e Gestão, Rio de Janeiro.

Sloterdijk, Peter

2002 Bulles. Sphères I, Paris, Pauvert.

Zask, Joëlle

2008 "Questions environnementales et participation démocratique », Raison publique, ${ }^{\circ} 8$, avril 2008, pp. 43-55, <http://www.raison-publique.fr/IMG/pdf/ raison_publique_o8.pdf $>$. 